ИЗВЕСТИЯ АКАДЕМИИ НАУК ЭСТОНСКОП ССР. ТОМ 28 ФИЗИКА * МАТЕМАТИКА. 1979, № 2

\title{
К РАСЧЕТУ ТУРБУЛЕНТНЫХ ТЕЧЕНИЙ В КАНАЛАХ И ТРУБАХ
}

\author{
(Представлена И. Эпиком)
}

Как известно, при описании развитого турбулентного течения в каналах и трубах принято условно различать две основные области - пристенный слой и ядро течения $\left[{ }^{1-3}\right]$. Профиль осредненной скорости в ядре течения обычно описывается параболической зависимостью. Для пристенного слоя (но за пределами зоны с преобладающим или заметным влиянием молекулярной вязкости) характерен логарифмический закон распределения скорости, который может быть выведен на основе различных теоретических соображений, включая использование простейших полуэмпирических моделей турбулентности или же анализа размерностей. Однако попытки получения такими способами единого для всей области развитого турбулентного течения аналитического выражения для профиля скорости (напр., в [ $\left.{ }^{4}\right]$ ) обычно не оказывались удачными. С другой стороны, использование для расчета осредненных и турбулентных характеристик течения уравнения баланса турбулентной энергии в его классической форме, требуя ряд дополнительных допущений, не позволяет получить искомые распределения в аналитическом виде $\left[{ }^{5,6}\right]$. Между тем, как будет показано ниже, сравнительно простое решение задачи с хорошей степенью точности может быть получено на основе феноменологической модели турбулентности, предложенной B $\left[{ }^{7}\right]$.

Для полностью установившегося турбулентного течения в плоском канале или круглой трубе постоянного сечения вне вязкого подслоя и в отсутствие массовых сил уравнения баланса импульса и баланса турбулентной энергии первичной составляющей пульсации скорости $\left[{ }^{7}\right]$ имеют следующий вид (в предположении $\partial\left(\overline{\left.u_{0} p / \varrho\right)} / \partial x \equiv 0\right.$, ось $x$ совмещена с осью течения):

$$
\begin{gathered}
-\frac{1}{\varrho} \frac{d P}{d x}+\frac{1}{y^{j}} \frac{d}{d y}\left[y^{j} N q_{0} l\left(\frac{d U}{d y}\right)\right]=0, \\
\frac{1}{y^{j}} \frac{d}{d y}\left[y^{j} N q_{0} l\left(\frac{d q_{0}^{2}}{d y}\right)\right]=0
\end{gathered}
$$

Здесь $U$ - осредненная скорость течения; $q_{0}$ - модуль вектора первичной составляющей пульсации скорости; $l$ - линейный масштаб турбулентности; $d P / d x$ - напорный градиент давления; @ - плотность; $N(y)$ - функция, связанная с распределением плотности вероятности пульсации скорости в различных направлениях; $j=0$ для плоского течения (канал), $j=1$ для осесимметричного течения (труба). 
Уравнения (1), (2) дополняются граничными условиями:

$$
U=U_{m}, q_{0}=q_{0 m}, d U / d y=d q_{0}^{2} / d y=0 \text { при } y=0 .
$$

Из уравнения (2) с учетом (3) следует

$$
q_{0}=q_{0 m}=\text { const }
$$

по всей ширине зоны развитого турбулентного течения.

В то же время использование уравнения, описывающего продольный перенос турбулентной энергии пульсациями давления $p\left[{ }^{7}\right]$

$$
\frac{\partial}{\partial x}\left(\overline{\left.u_{0} p / \varrho\right)}=-B \frac{q_{0}^{3}}{l}+C\left(\frac{1}{\varrho}\left|\frac{d P}{d x}\right| a\right)^{3 / 2} / l\right.
$$

( $a$ - полуширина канала или радиус трубы, $B, C-$ константы), при $\partial\left(\overline{u_{0} p} / \varrho\right) / \partial x \equiv 0$ и с учетом равенства

$$
\frac{1}{\varrho} \frac{d P}{d x}=(j+1) \frac{\tau_{w}}{\varrho a}=(j+1) \frac{U_{*}^{2}}{a}
$$

( $\tau_{w}$ - касательное напряжение на стенке, $U$. - скорость трения), дает

$$
q_{0}=(C / B)^{1 / 3} U *(j+1)^{1 / 2}=\text { const или } \quad q_{0 m} / U_{*}=(C / B)^{1 / 3}(j+1)^{1 / 2} .
$$

Равенство (7) описывает в рамках модели $\left[{ }^{7}\right]$ локальный баланс турбулентной энергии (порождение равно диссипации) в установившемся течении. В отличие от традиционного выражения для локального баланса энергии равенство (7) является точным, так как, согласно (2), диффузия энергии заведомо отсутствует.

Положим

$$
N=\left[1-(y / a)^{n}\right] / 3,
$$

где $n-$ константа. Поскольку в выражении для турбулентной вязкости

$$
v_{t}=N q_{0} l \text {, }
$$

$q_{0}=$ const (согласно (4)) и $l=$ const (что вытекает из принятого в $\left[{ }^{7}\right]$ допущения $l / a=$ const в любом турбулентном пограничном слое), то $v_{t} \sim N$. Согласно $(8)$, при достаточно большом $n$ турбулентная вязкость остается почти постоянной на значительном участке ядра течения, причем на оси течения предполагается равная вероятность турбулентных пульсаций скорости в любом направлении (т. е. $N_{m}=1 / 3$ ). Вблизи же стенки $\left(y^{\prime}=a-y \ll a\right)$ уравнение (8) (если заменить в нем $y$ на $y^{\prime}$, разложить полученное выражение в ряд Маклорена и сохранить только первые два члена разложения) сводится к зависимости

$$
N=n\left(y^{\prime} / a\right) / 3,
$$

что соответствует линейному закону возрастания турбулентной вязкости вблизи стенки.

С учетом соотношений $(3),(4),(6)$ и (7) получим из (1)

$$
d(U / U \cdot) / d(y / a)=A(y / a) /\left[1-(y / a)^{n}\right],
$$

где $A=3\left(U \cdot / q_{0 m}\right)(a / l)$. 


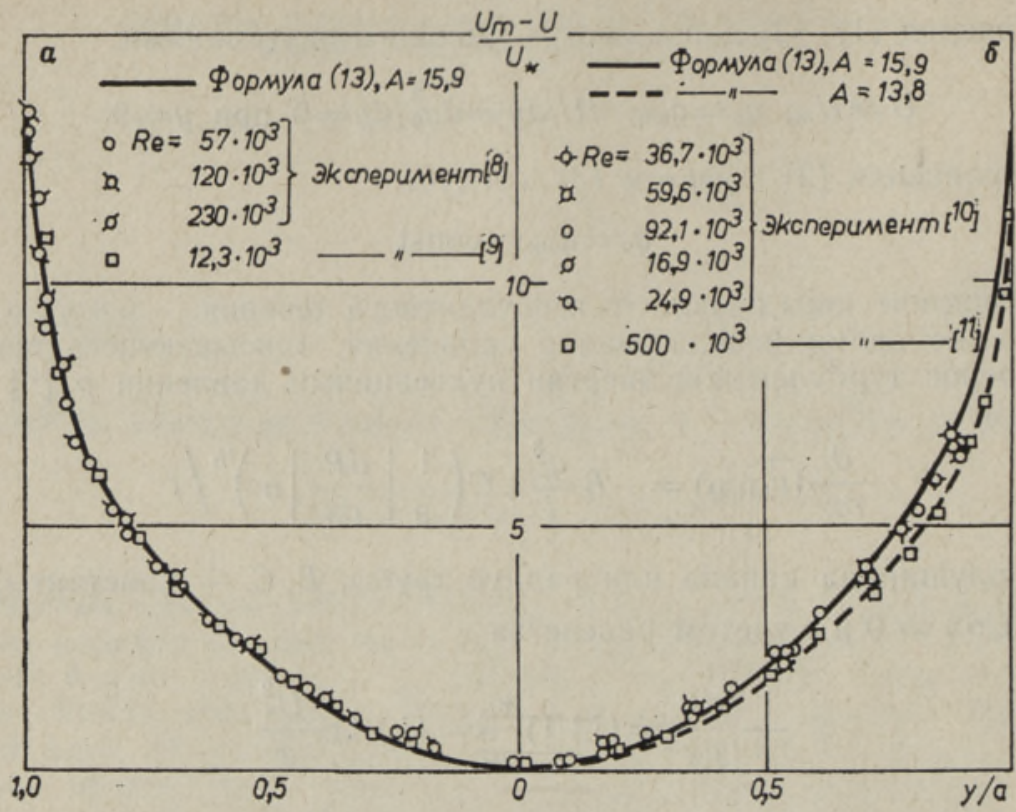

Рис. 1.

Таким образом, принятая модель приводит к одному и тому же профилю осредненной скорости для плоского и осесимметричного течений.

Вблизи оси течения $(y / a \ll 1)$ интегрирование уравнения (9) приводит к параболической зависимости

$$
\left(U_{m}-U\right) / U_{*}=A(y / a)^{2} / 2,
$$

а вблизи стенки $\left(y^{\prime} / a \ll 1\right)-$ к логарифмическому закону

$$
\left(U_{m}-U\right) / U \cdot=-\ln \left(y^{\prime} / a\right) / K+K_{1},
$$

где $K=n / A$ и $K_{1}-$ константы.

При $n=8$ интегрирование (10) дает следующую аналитическую зависимость для профиля дефицита скорости по всей зоне развитого турбулентного течения:

$$
\left(U_{m}-U\right) / U_{*}=A\left[\operatorname{Arth}(y / a)^{2}+\operatorname{arctg}(y / a)^{2}\right] / 4 .
$$

Сравнение расчета по (13) с опытными данными показано на рис. 1 ( $a$ - канал, 6 - труба). Как видно, их хорошее совпадение для течения в канале $\left[{ }^{8,9}\right]$ и для течения в трубе $\left[{ }^{10}\right]$ достигается при $A=15,9$, в то время как опытным данным [11] для трубного течения лучше удовлетворяет значение $A=13,8$. При $A=15,9$ и $n=8$ получим для константы $K$ логарифмического профиля (12) величину 0,50 , существенно превышающую общепринятое значение константы Кармана 0,40. Остановимся на этом вопросе подробнее.

Отметим прежде всего, что логарифмический закон (12) для напорного течения в канале или трубе является лишь аппроксимацией, тем более справедливой, чем точнее выполняется условие постоянства касательных напряжений $(\tau=$ const), т. е. по мере приближения к стенке (но за пределами вязкого слоя). Анализ различных опытных данных 
Рис. 2.

по установившимся течениям в каналах и трубах $\left[{ }^{8-11}\right]$ дает значения $K$ в пределах от 0,33 до 0,41 . В сущности, если учитывать естественный разброс опытных данных, численное значение $K$ будет зависеть от требуемой точности, с которой должен выполняться логарифмический закон. Стремление «подогнать» под этот закон как можно больше опытных точек по направлению к оси течения приводит к заниженным значениям $K$. И, напро-

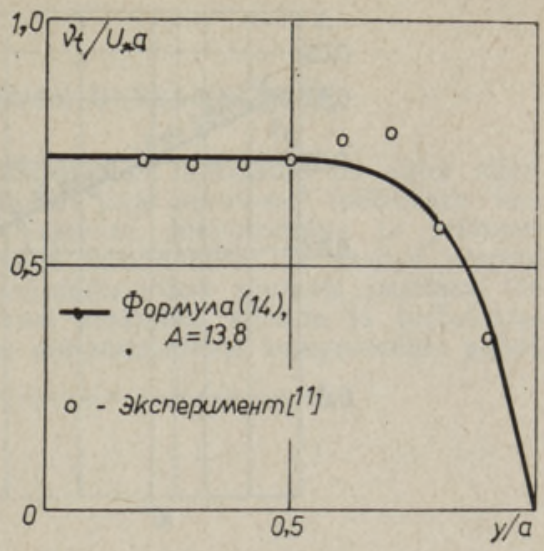
тив, более высокие значения $K$ свидетельствуют лишь о том, что логарифмический закон справедлив фактически на меньших расстояниях от стенки, чем это обычно принято считать. В данной работе численные значения константы $A$ (а следовательно, и $K$ ) подбирались так, чтобы наилучшим образом удовлетворить опытным данным не только вблизи стенки, а по всей ширине зоны развитого турбулентного течения. Кроме того, численное значение $K$ зависит также от экспоненты $n$ в формуле (8). Так, при $A=15,9$ и $n=6$ получим $K=0,38$; однако в этом случае расчет согласуется с опытными данными в целом гораздо хуже, чем при $n=8$. С другой стороны, опытным данным [ $\left.{ }^{11}\right]$ при $A=13,8$ наилучшим образом удовлетворяет значение $n=7$ (а не $n=8$ ), но в этом случае снова получаем $K \cong 0,50$. Таким образом, говорить о каком-то фактическом значении константы $K$ можно лишь весьма условно.

Подставляя (8) в (9) и учитывая (7), получим зависимость

$$
v_{t}=\left[1-(y / a)^{n}\right] a U \cdot / A,
$$

которая при $y^{\prime} / a=1-y / a \ll 1$ сводится к известному закону $\left[{ }^{1-3}\right]$

$$
v_{t}=K U * y^{\prime} \text {. }
$$

Сравнение расчета по формуле (14) (при $A=13,8$ и $n=8$ ) с опытными данными $\left[{ }^{11}\right]$ показано на рис. 2.

Выведем также зависимость для коэффициента сопротивления $\lambda=$ $=8\left(U \cdot / U_{\mathrm{c}}\right)^{2}$ при течении в гладких трубах. Средняя по сечению скорость, определенная по уравнению (13) при $A=15,9$, равна

$$
U_{\mathrm{c}}=U_{m}-4,5 U_{*}
$$

Связь между $U_{m}$ и $U$. в зависимости от числа $\operatorname{Re}=U_{\mathrm{c}} a / v$ находим, используя классическую двухслойную схему течения [12] и учитывая, что на условной границе ламинарного подслоя $y^{\prime} / a=1-\left(y_{\text {л }} / a\right)=$ $=\alpha(v / a U$.$) скорость равна U=\alpha U$. Здесь $5<\alpha<30$. где нижний предел характеризует толщину вязкого подслоя, а верхний - толщину буферного слоя. Полагая $\alpha=13$ и имея в виду, что $\operatorname{Arth}[1-\alpha(v / a U \text {. })]^{2} \gg$ $\gg \operatorname{arctg}\left[1-\alpha\left(v / a U_{*}\right)\right]^{2}$ на расстоянии $y_{\text {л }}^{\prime} / a$, получим следующее уравнение для коэффициента сопротивления:

$$
1 / \sqrt{\lambda}=0,35\left[8,5+2,0 \operatorname{Arth}(1-36,8 / \operatorname{Re} \sqrt{\lambda})^{2}\right] .
$$




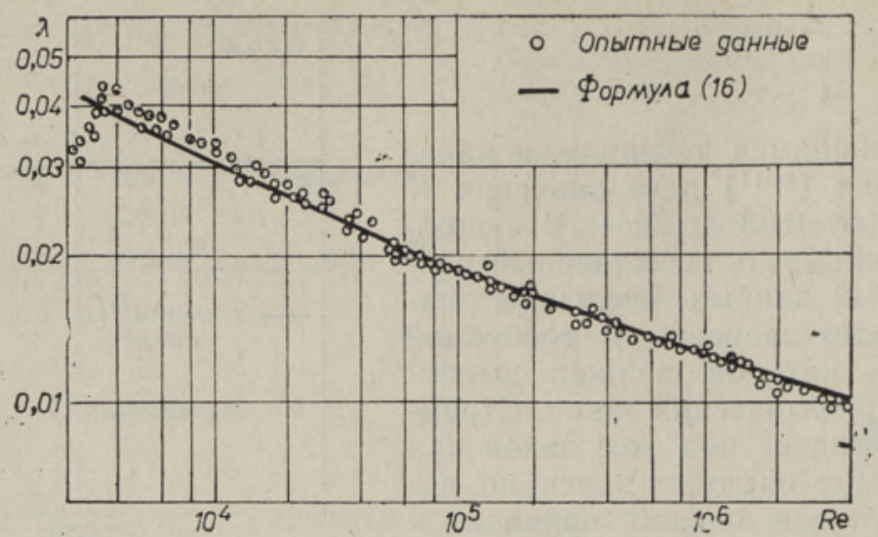

Рис. 3.

Сравнение расчета по формуле (16) с опытными данными разных авторов $\left[{ }^{12}\right]$ показано на рис. 3 .

Входящие в уравнение (5) константы $B$ и $C$ могут быть определены на основе опытных данных по измерению скорости диссипации энергии $\varepsilon$ и с помощью формулы (7). Такие измерения в круглой трубе дали на оси течения значения $\varepsilon_{m} a / U_{*}^{3}$, равные 2,4 в $\left[{ }^{10}\right]$ и 1,5 в [ $\left.{ }^{11}\right]$. В опытах $[8,10,11]$ для течений в каналах и трубах было получено примерно одинаковое значение $q_{0 \mathrm{~m}} / U_{*} \approx 1,4$. Тогда при $K=15,9$ верно $l / a=0,135$. Принимая величину $\varepsilon_{m} a / U_{*}^{3}=2,4$ как более надежную и учитывая, что согласно модели $\left[{ }^{7}\right]$ на оси течения имеет место равенство $q_{\alpha^{2}}=\overline{q^{2}}$ $\left(\overline{q^{2}}-\right.$ полная кинетическая энергия турбулентности), находим из (7) $B \approx 0,12$ и $C \approx 0,12$. Такие значения констант могут быть приняты при расчете течений в круглых трубах на участке установления.

\section{ЛИТЕРАТ У РА}

1. Монин А. С., Яглом А. М., Статистическая гидромеханика, ч. I, М., «Наука», 1965.

2. Хи нц е И. О., Турбулентность, М., Физматгиз, 1963.

3. Те пакс Л., Равномерное турбулентное движение в трубах и каналах, Таллин, «Валгус», 1975.

4. Gos s e, J., C. r. Acad. sci., 253, № 1, 61-63 (1961).

5. В а гер Б. Г., Л а йхтм ан Д. Л., Изв. АН СССР, Мех. жидкости и газа, № 4, $89-92$ (19368).

6. Ак а тн ов Н. И., Т у льв е р т В. Ф., Изв. АН СССР, Мех. жидкости и газа, № 3 , $25-33$ (1973)'

7. Э п ш т е й н А., Изв. АН ЭССР, Физ. Матем., 27, № 4ı 4113-422 (1978).

8. Кон т-Белло Ж., Турбулентное течение в канале с параллельными стенками, М., «Мир», 1968.

9. L a u f e r, J., Investigation of turbulent flow in a two-dimensional channel, NACA Rep. 1053, 1951.

10. L a w n, C. J., J. Fluid Mech., 48, № 3, 477-505 (1971).

11. L a u f e r, J., The structure of turbulence in fully developed pipe flow, NACA Rep. $1174,1954$.

12. Ш лих ти н г Г., Теория пограничного слоя, М., «Наука», 1974.

Ннститут термофизики и электрофизики Академии наук Эстонской ССР
Поступила в редакцию 28/VI 1978 


\section{A. EPSTEIN}

\section{MÓNEDEST VABA TURBULENTSUSE ULESANNETEST}

Oma varem avaldatud fenomenoloogilist turbulentsusmudelit kasutades on autor käesolevas artiklis esitanud kahemõōtmelise joa ja silindri jälje arvutused (põhiosas). Isemodelleeruvatesse muutujatesse transformeeritud pidevuse, liikumishulga ja primaarkomponentide turbulentse energia tasakaaluvõrrandite lahendamisel on saadud eespool mainitud juga iseloomustavate keskmistatud ja pulsatsiooniliste suuruste jaotused. On märgitud arvutuste ja katseandmete head vastavust keskmiste kiiruste ja turbulentse nihkepinge korral ning mõningast hajumist kiiruse pulsatsiooniliste komponentide puhul, samuti käsitletud hajumise võimalikke põhjusi.

\section{A. EPSTEIN}

\section{CALCULATION OF TURBULENT CHANNEL AND PIPE FLOWS}

Calculation of fully developed uniform turbulent flow of incompressible fluid in a plane channel or round pipe is carried out on the basis of a phenomenological model of turbulence proposed in $\left[^{7}\right]$. A single formula (12) for the mean velocity defect profile in both channel and pipe flows has been derived as the result of an analytical solution of the momentum and turbulent energy equations (1), (2) with appropriate boundary conditions (3) and some additional assumptions. Near the wall the profile (12) tends to the well-known logarithmic law (11) and near the flow axis - to the parabolic one (10). The calculated velocity defect profile agrees quite well with the experimental data of Conte-Bellot $\left[{ }^{8}\right]$ and Laufer $\left[{ }^{9}\right]$ for the channel flow as well as the results of Lawn $\left[{ }^{10}\right]$ and Laufer $\left[{ }^{11}\right]$ for the pipe flow (Fig. 1).

An analytical expression for turbulent viscosity (13) is compared with the experimental data of Laufer [ $\left.{ }^{11}\right]$ (Fig. 2). Also a relation for the friction coefficient in pipe flow as a function of the Reynolds number (15) is derived, in good agreement with the experimental data reported by various investigators (Fig. 3). 